\title{
Determination of Local Chemistry Composition of Low-Dimensional Semiconductor Nanostructures Through the use of High-Resolution HAADF images
}

\author{
D. Hernández-Maldonado ${ }^{1,2,3}$, M. Herrera ${ }^{1}$, A.R. Lupini ${ }^{4}$, S. I. Molina ${ }^{1}$ \\ ${ }^{1 .}$ Departamento de Ciencia de los Materiales e I.M. y Q.I., Facultad de Ciencias, \\ Universidad de Cádiz, Campus Río San Pedro, s/n, 11510 Puerto Real, Cádiz, Spain \\ ${ }^{2}$ SuperSTEM Laboratory, STFC Daresbury Campus, Daresbury WA4 4AD, UK \\ ${ }^{3}$.Department of Materials, University of Oxford, Parks Road, Oxford OX1 3PH, UK \\ ${ }^{4}$ Materials Science and Technology Division, Oak Ridge National Laboratory, Oak Ridge, \\ TN 37831, USA
}

The introduction of aberration-correction into Scanning Transmission Electron Microscopy (STEM) has vastly improved the ability to carry out chemical analysis of low-dimensional semiconductor nanostructures, which is a topic of high relevance in fields such as electronics and optoelectronics [1,2]. Among different techniques in STEM, Energy Electron Loss Spectroscopy (EELS) and Energy-dispersive X-ray Spectroscopy (EDX) are currently the most used to obtain a quantitative map of the structures' chemical composition. However, in practice, extracting information from an area of the size of a Quantum Dot (QD) with these techniques in STEM mode is a complicated task, due to the time necessary to record the signal. During this time drift effects or sample damage could occur. Thus a method that uses only the High Angle Annular Dark Field (HAADF) signal to analyse the composition could avoid these problems, because in a few seconds it is possible to image large areas.

HAADF images of QDs have been widely used for a qualitative analysis of their chemical distribution and for morphological characterization [3]. However, due to the high dependence of the intensity with the atomic number $(Z)$ of the HAADF images, the extraction of quantitative information from this sort of image has become a topic of great interest. Nowadays there are multiple options to interpret HAADF images in quantitative terms. It is possible to study the intensities associated with atomic columns through statistical analysis [4], phenomenological procedures [5] or to compare with simulations [6]. But in the case of the analysis of nanostructures like QDs all of these methodologies are affected by the dependence of the HAADF intensities on the strain fields originating from the nanostructure that alter the intensities [7], making the precise quantification of HAADF images a complicated task.

This paper presents an alternative method to quantify the chemical composition of low dimensional nanostructures based on the analysis of the atomic column positions present in High-Resolution HAADF images. These atomic positions depend on the strain field of the nanostructure, which is closely related to the local composition of the structure. By a comparison between the strain maps measured from experimental HR-HAADF images and the strain maps obtained by Finite Element Method (FEM) applied to a model that describes the nanostructure, it is possible to determine the local chemical composition. We have applied this methodology to a nanostructure formed by two vertically-stacked InGaAs QDs. Figure 1a) is a HAADF image of the nanostructure used as an example. Bright areas are associated with the presence of In atoms. Images like this have been employed to construct a three-dimensional (3D) model where FEM calculations have been applied. Figure 1b) is the 3D model proposed for the structure under study, which is composed of different parts. During the process of comparison, the chemical concentration of each part of the model is 
varied in a repetitive procedure until a good fit between the experimental and the calculated strain map is obtained. Figure 2a) is the experimental strain map of the nanostructure parallel to the growth direction and $2 b$ ) is the corresponding map calculated by FEM obtained after the fitting process. As a result of the process we obtain a composition profile along the nanostructure studied. Some considerations in the interpretation of the results, due to the fact that the depth of field of an Aberration-Corrected STEM is less than the thickness of the sample under study will be discussed.

\section{References:}

[1] G. Biasiol, et al. Physics Reports 500 (2011) 117-173.

[2] S. Kadkhodazadeh. Micron 44 (2013) 75-92.

[3] P. Wang, et al. Applied Physics Letters 89 (2006)072111.

[4] S. Van Aert, et al. Physical Review B 87 (2013) 064107.

[5] S. I. Molina, et atl. Ultramicroscopy 109 (2009) 172-176.

[6] P.L. Galindo. Journal of Physics 522 (2014) 012013.

[7] T. Grieb. Ultramicroscopy 129 (2013) 1-9.

[8] This research was supported by the Spanish MINECO (project TEC2011-29120-C05-03 and CONSOLIDER INGENIO CSD2009-00013) and the Junta de Andalucía (PAI research group TEP-946 INNANOMAT). Co-funding from UE is also acknowledged. Work at Oak Ridge National Laboratory was sponsored by the U.S. Department of Energy, Division of Materials Sciences and Engineering. We thank L. Gonzalez and Y. Gonzalez from IMM for supplying the sample.

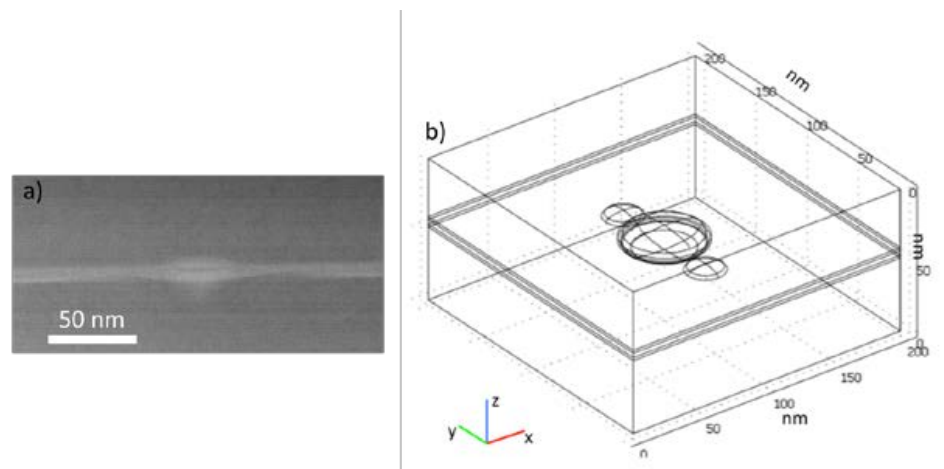

Figure 1a) HAADF image of the nanostructure studied, which is formed by 2 QDs vertically stacked. b) Three-dimensional model of the previous structure created for the FEM calculations.

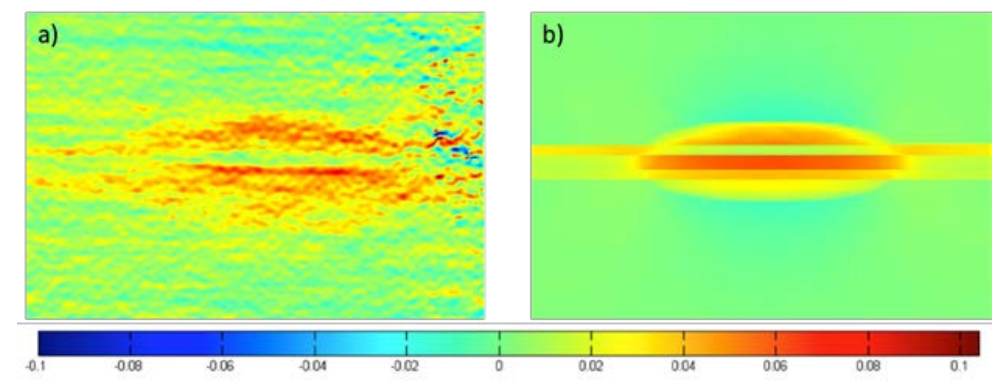

Figure 2 a) Strain map obtained from the Geometrical Phase Analysis of a HR-HAADF image of the nanostructure presented in figure 1a). b) Calculated strain map of the 3D model proposed for the nanostructure. 